\title{
The usefulness of radiographic imaging for predicting the resectability of thymic carcinoma
}

\author{
Yoshiyuki Ozawa \\ Department of Radiology, Nagoya City University, Graduate School of Medical Sciences, Mizuho-ku, Nagoya, Japan \\ Correspondence to: Yoshiyuki Ozawa, MD, PhD. Department of Radiology, Nagoya City University, Graduate School of Medical Sciences, 1 \\ Kawasumi, Mizuho-cho, Mizuho-ku, Nagoya 467-8601, Japan. Email: ykiooster@gmail.com. \\ Provenance: This is an invited Editorial commissioned by Executive Editor-in-Chief Jianxing He (Director of the Thoracic Surgery Department, The \\ First Affiliated Hospital of Guangzhou Medical University, Guangzhou, China). \\ Comment on: Hayes SA, Huang J, Golia Pernicka J, et al. Radiographic Predictors of Resectability in Thymic Carcinoma. Ann Thorac Surg \\ 2018;106:242-8.
}

Submitted Aug 23, 2018. Accepted for publication Sep 13, 2018.

doi: $10.21037 /$ jtd.2018.09.88

View this article at: http://dx.doi.org/10.21037/jtd.2018.09.88

Thymic carcinoma is a rare type of thymic epithelial malignancy, and the optimal treatment for this disease has not been established, due to the limited amount of data available. However, surgery plays a central role in the management of such tumors. Hayes et al. (1) reported computed tomography (CT) findings that can be used to predict the resectability of thymic carcinoma. In other studies, the resection status and the Masaoka stage were identified as a prognostic factor for patients with thymic carcinoma that underwent surgical resection (2-4). R0 resection, complete macroscopic and microscopic resection, is an important prognostic factor in such cases and is associated with an improved overall survival rate (2-6). So, identifying preoperative factors that could be used to predict whether a thymic carcinoma can be completely resected would help physicians to predict the prognosis of patients with such tumors and/or help them to determine which patients would benefit from neoadjuvant therapy. A study by Hayes et al. focusing on CT-based predictors of the resectability of thymic carcinoma (1) evaluated many preoperative imaging features, including tumor size, CT attenuation values, tumor contours, the presence/absence of tumor calcification, pulmonary nodules, lymphadenopathy, pleural effusion/nodularity, and distant metastases. Additionally, it looked at the relationship between the tumor and the adjacent structures. In the latter study, the following CT features were found to be significantly associated with an incomplete resection, the degree of contact between the tumor and adjacent blood vessels and the maximum tumor size. In total, $85 \%$ of cases in which the resection was incomplete, had involved tumors that were in contact with $>25 \%$ of an adjacent mediastinal structure on pre-operative CT, whereas this was only the case in $45 \%$ of the cases in which complete resection was achieved. A maximum tumor size cut-off value of $>7.5 \mathrm{~cm}$ resulted in sensitivity, specificity, and area under curve (AUC) values for predicting incomplete resection of $0.55,0.905$, and 0.737 , respectively. In addition, tumor invasion into the mediastinal vascular structures, was identified as a major cause of an incomplete surgical resection. However, CT findings regarding the degree of which a tumor is in contact with adjacent structures can be a little ambiguous, and their interpretation can vary among clinicians. The sample size of the aforementioned study was also small, partly because of the rarity of thymic carcinoma, so the possibility of an undetectable statistically significant difference in other CT findings cannot be dismissed.

As mentioned above, preoperatively estimating the resectability of thymic carcinoma is important for managing it. CT is a relatively non-invasive form of examination, and provides information about various factors, such as a tumor's location and size, the relationship between the tumor and the surrounding structures, and the presence or absence of a distance metastasis. So, CT plays a central role in preoperative evaluations of thymic carcinoma. However, as various methods can be used to interpret substantial data 
of CT, more accurate and objective evaluation methods would be preferable. It is still challenging to interpret whether tumors, including thymic epithelial tumors, have invaded the adjacent organs on a CT scan. The following factors are examined when trying to determine whether a tumor has invaded the adjacent blood vessels: the presence or absence of fat tissue separating the tumor from the nearby blood vessels, the proportion of the circumference of a blood vessel that is in contact with the tumor (usually a cut-off value of $>50 \%$ of the blood vessel circumference is used), the presence/absence of tumor-induced blood vessel compression, deformation, and/or occlusion, or an irregular border between the tumor and an adjacent blood vessel (7-10). The presence or absence of a tumor-induced blood vessel compression, deformation, and/or occlusion and whether the tumor is in direct contact with more than half of the circumference of a blood vessel are CT features that result in a relatively good diagnostic accuracy, when assessing a blood vessel invasion (8). However, the absence of fat tissue separating a tumor from the adjacent blood vessels on a CT scan, cannot be used to definitively diagnose blood vessel invasion, although the presence of such fat is important for excluding such invasion. Multiplanar reconstruction (MPR) can be useful for further evaluating tumor invasion because accurate depiction of the borders between the tumors, and adjacent structures, depends on the planar direction of the images. Curved MPR is especially useful for diagnosing invasion into serpentine or curved blood vessels, because it produces cross-sectional images along the length of blood vessels, which facilitates evaluations of intraluminal abnormalities and the borders between tumors and the adjacent blood vessels. A slice thickness of $5 \mathrm{~mm}$ might be too thick for evaluating a tumor invasion into adjacent structures. The use of thinner slices, reduces partial volume effects, and might help to improve the accuracy of the assessments of tumor invasion.

There have also been some studies in which the dynamic imaging of the cardiothoracic region has been used to analyze the motion of tumors and the adjacent structures. Four-dimensional (4D)-CT or cine-magnetic resonance imaging (MRI) have also been used to improve diagnostic accuracy in such cases, especially to visualize the motion derived from respiration, or the pulsation of cardiovascular structures (10-14). Both the CT and the MRI, have been reported to be useful for diagnosing tumor invasion; however, it is difficult to assess tumor invasion into adjacent blood vessels on a non-contrast-enhanced CT. While simultaneously, the non-contrast-enhanced MRI is useful for evaluating patients with renal dysfunction, or those that are allergic to iodine-based contrast medium. It has been reported that assessing the sliding motion of tumors and the adjacent blood vessels during dynamic scans improves the accuracy of preoperative evaluations in terms of their ability to determine the presence or absence of a tumor invasion into adjacent structures. It has been suggested that a minimal motion of cardiovascular structures, such as the distal aortic arch, descending thoracic aorta, superior vena cava (SVC), and pulmonary veins, could increase the risk of a false-positive diagnosis of cardiovascular invasion by a thoracic mass when analyzing the sliding motion of tumors (14). Anterior mediastinal tumors, including thymic carcinomas, are often in direct contact with blood vessels, such as the SVC, left brachiocephalic vein, aorta, and pulmonary artery, as well as the pericardium and pulmonary parenchyma. So, assessments of a tumor invasion into blood vessels that move minimally, should be done carefully. Changes in body positions that separate tumors from the adjacent structures might help to solve such problems. Four dimension-CTs and cine-MRIs provide a lot of additional information for assessing tumor invasion, as mentioned above. In addition, determining the locations of any invaded structures using such imaging would provide useful preoperative information for surgeons.

Improvements in the preoperative evaluations of CT images of patients with thymic carcinoma would result in a more precise therapeutic plan. Further studies that are aimed at developing methods to allow a more accurate prediction of the resectability of thymic carcinoma are desired.

\section{Acknowledgements}

None.

\section{Footnote}

Conflicts of Interest: The author has no conflicts of interest to declare.

\section{References}

1. Hayes SA, Huang J, Golia Pernicka J, et al. Radiographic predictors of resectability in thymic carcinoma. Ann Thorac Surg 2018;106:242-8.

2. Hishida T, Nomura S, Yano M, et al. Long-term outcome and prognostic factors of surgically treated thymic 
carcinoma: results of 306 cases from a Japanese Nationwide Database Study. Eur J Cardiothorac Surg 2016;49:835-41.

3. Weksler B, Dhupar R, Parikh V, et al. Thymic carcinoma: a multivariate analysis of factors predictive of survival in 290 patients. Ann Thorac Surg 2013;95:299-303.

4. Ruffini E, Detterbeck F, Van Raemdonck D, et al. Thymic carcinoma: a cohort study of patients from the European society of thoracic surgeons database. J Thorac Oncol 2014;9:541-8.

5. Filosso PL, Guerrera F, Rendina AE, et al. Outcome of surgically resected thymic carcinoma: a multicenter experience. Lung Cancer 2014;83:205-10.

6. Ahmad U, Yao X, Detterbeck F, et al. Thymic carcinoma outcomes and prognosis: results of an international analysis. J Thorac Cardiovasc Surg 2015;149:95-100, 101.e1-2 .

7. Marom EM, Milito MA, Moran CA, et al. Computed tomography findings predicting invasiveness of thymoma. J Thorac Oncol 2011;6:1274-81.

8. Shen Y, Ye J, Fang W, et al. Efficacy of computed tomography features in predicting stage III thymic tumors. Oncol Lett 2017;13:29-36.

9. Ozawa Y, Hara M, Shimohira M, et al. Associations between computed tomography features of thymomas and their pathological classification. Acta Radiol 2016;57:1318-25.

Cite this article as: Ozawa Y. The usefulness of radiographic imaging for predicting the resectability of thymic carcinoma. J Thorac Dis 2018;10(Suppl 33):S3970-S3972. doi: 10.21037/ jtd.2018.09.88
10. Lee CH, Goo JM, Kim YT, et al. The clinical feasibility of using non-breath-hold real-time MR-echo imaging for the evaluation of mediastinal and chest wall tumor invasion. Korean J Radiol 2010;11:37-45.

11. Hong YJ, Hur J, Lee HJ, et al. Respiratory dynamic magnetic resonance imaging for determining aortic invasion of thoracic neoplasms. J Thorac Cardiovasc Surg 2014;148:644-50.

12. Choong CK, Pasricha SS, Li X, et al. Dynamic fourdimensional computed tomography for preoperative assessment of lung cancer invasion into adjacent structures $†$. Eur J Cardiothorac Surg 2015;47:239-43; discussion 243.

13. Wang L, Lv P, Yang S, et al. Assessment of thoracic vasculature in patients with central bronchogenic carcinoma by unenhanced magnetic resonance angiography: comparison between 2D free-breathing TrueFISP, 2D breath-hold TrueFISP and 3D respiratorytriggered SPACE. J Thorac Dis 2017;9:1624-33.

14. Seo JS, Kim YJ, Choi BW, et al. Usefulness of magnetic resonance imaging for evaluation of cardiovascular invasion: evaluation of sliding motion between thoracic mass and adjacent structures on cine MR images. J Magn Reson Imaging 2005;22:234-41. 